\title{
Purification and characterisation of lactate dehydrogenase: an undergraduate biochemistry laboratory experiment
}

\author{
Yannis Karamanos \\ Laboratoire de Biochimie, Faculté des Sciences Université d'Artois, Lens, France \\ Email address: \\ yannis.karamanos@univ-artois.fr
}

To cite this article:

Yannis Karamanos. Purification and Characterisation of Lactate Dehydrogenase: An Undergraduate Biochemistry Laboratory Experiment. Advances in Biochemistry. Vol. 2, No. 1, 2014, pp. 14-23. doi: 10.11648/j.ab.20140201.13

\begin{abstract}
The practical work described here was designed in the aim of combining several periods that were previously carried-out independently during the academic year and to more appropriately mimic a "research" environment. It illustrates several fundamental biochemical principles as well as experimental aspects and important techniques including spectrophotometry, chromatography, centrifugation, and electrophoresis. Lactate dehydrogenase (LDH) is an enzyme of choice for a student laboratory experiment. This enzyme has many advantages, namely its relative high abundance, high specific activity and high stability. In the first part, the purification scheme starting from pig heart includes ammonium sulphate fractionation, desalting by size exclusion chromatography, anion exchange chromatography and pseudo-affinity chromatography. In the second part of the work the obtained fractions are accessed for protein and activity content in order to evaluate the efficiency of the different purification steps, and are also characterised by electrophoresis using non-denaturing and denaturing conditions. Finally, in the third part, the purified enzyme is subjected to comprehensive analysis of its kinetic properties and compared to those of a commercial skeletal muscle LDH preparation. The results presented thereafter are representative of the data-sets obtained by the student-pairs and are comparable to those obtained by the instructors and the reference publications. This multistep purification of an enzyme from its source material, where students perform different purification techniques over successive laboratory days, the characterisation of the purified enzyme, and the extensive approach of enzyme kinetics, naturally fits into a project-based biochemistry learning process.
\end{abstract}

Keywords: Lactate Dehydrogenase, LDH, Protein Purification, Enzymes, Laboratory Experiment

\section{Introduction}

The project of this practical work was initiated by the author at Limoges University in the early 90's in the aim of combining several laboratory periods (3-4 h each) that were previously carried-out independently during the academic year and to more appropriately mimic a "research" environment. The project was continuously updated to integrate recent tools, the purification steps were scaled-down and the organisation was optimized. The current "built-in" biochemistry laboratory experiment is designed for the last-year of the bachelor degree students (the third-year of "licence" in the French context). In this way it became possible, in a linked series of laboratories, to illustrate several fundamental biochemical principles as well as experimental aspects and important techniques including spectrophotometry, chromatography, centrifugation, and electrophoresis. By the way the students become the central actors of the project and finally they have a better understanding of the constraints in a laboratory. They have to prepare themselves all the buffers and reagents and to be aware of all the safety rules. Lactate dehydrogenase (LDH) is an enzyme of choice for a student laboratory experiment. This enzyme has many advantages, namely its relative high abundance in heart and muscle that could be used for the purification, high specific activity and high stability. The experimental work is organised in three parts. In the first part the students have to purify LDH from pig heart, but this source could be replaced by others. The purification utilizes several steps namely fractionation by ammonium sulphate (AS) precipitation, desalting by size exclusion chromatography, anion exchange chromatography and pseudo-affinity chromatography. The purification scheme was inspired and adapted from previously published procedures [1,2]. In the second part of the work the obtained 
fractions are accessed for protein and activity content in order to evaluate the efficiency of the different purification steps. The fractions are also characterised by electrophoresis under non-denaturing and denaturing conditions. Finally, in the third part, the purified enzyme is subjected to analysis of its kinetic properties and compared to those of a commercial skeletal muscle LDH preparation.

\section{Background and Student Preparation}

\subsection{Background}

LDH (EC 1.1.1.27; L-lactate: NAD ${ }^{+}$oxiroreductase) is a ubiquitous tetrameric enzyme among vertebrates organisms that performs the following equilibrated reaction:

$$
\mathrm{NAD}^{+}+\text {Lactate } \rightleftharpoons \mathrm{NADH}+\mathrm{H}^{+}+\text {pyruvate }
$$

LDH is most often measured to check for tissue damage. It is present in many body tissues, especially the heart, liver, kidney, muscles, brain, blood cells, and lungs. Mammalian $\mathrm{LDH}$ exists as five isoenzymes - A4; A3B; A2B2; AB3; and $\mathrm{B} 4$ - that have distinct kinetics constants as well as $\mathrm{pH}$ and temperature responses [3]. The A4 isoenzyme is found in skeletal muscle and the B4 in heart muscle. The use of the $\mathrm{A}$ and $\mathrm{B}$ nomenclature for the subunits is preferred as an alternative to $\mathrm{M}$ and $\mathrm{H}$, to signify that the same isoenzymes of LDH are found in tissues other than muscle [4].

Its activity is typically measured at $340 \mathrm{~nm}\left(\mathrm{~A}_{340}\right)$ by following either the oxidation of $\mathrm{NADH}$ with pyruvate (decrease in absorbance) or reduction of $\mathrm{NAD}^{+}$with lactate (increase in absorbance). One unit (U) of the enzyme activity is generally defined as the amount of enzyme catalyzing the oxidation of $\mathrm{NADH}$ or the reduction of $\mathrm{NAD}+$ at a rate of $1 \mu \mathrm{mol}$ per min.

\subsection{Student Preparation}

Students have to be already instructed with basic enzyme kinetics, know the $\mathrm{LDH}$ reaction mechanism and the structures of all the substrates and reaction products. They have to participate in a pre-lab lecture and also have to read a handout containing the background information, the experimental section, and in particular how to prepare the buffers, ammonium sulphate precipitation and theory of electrophoresis. Among other skills they should be confident in spectrophotometry and the Beer-Lambert law, the preparation of solutions, the use of micro-pipettes.

\section{Experimental Section}

\subsection{Materials}

All chemicals were reagent grade or equivalent. DEAE-Sepharose CL-6B and Cibacron Blue 3GA-agarose were from Sigma-Aldrich and PD-10 disposable columns (Sephadex G25 gel) from GE Healthcare. ProteoStain was from Proteomics Consult, Belgium. The hearts were obtained at a local slaughterhouse from freshly slaughtered pigs, divided in several 60-70 g portions and kept frozen.

\subsection{Purification}

Preparation of Crude Extract. This step is carried out once for all the student-pairs. A piece of heart, slowly towed at $4{ }^{\circ} \mathrm{C}$, freed of fat, vessels and valves, and approximately $50 \mathrm{~g}$ are cut into strips and homogenized in a Waring Blendor with $100 \mathrm{~mL}$ of cold extraction buffer $(50 \mathrm{mM}$ Tris-HCl, pH 7.50, 1 mM EDTA, $1 \mathrm{mM} \beta$-mercaptoethanol) twice for $1 \mathrm{~min}$. The extract is centrifuged at $15000 \mathrm{x} g$ for $15 \mathrm{~min}\left(4^{\circ} \mathrm{C}\right)$ and the supernatant filtered through glass wool if necessary. This crude extract (fraction name: $\mathrm{CE}$ ) is dispatched to the student-pairs $(10 \mathrm{~mL}$ per group in a Falcon-type tube).

Ammonium Sulphate Fractionation. The extract is brought to $40 \%$ saturation by slowly adding solid ammonium sulphate (AS) with gentle stirring on ice. The suspension is then let on ice for at least $30 \mathrm{~min}$ and then centrifuged at $15000 \times \mathrm{g}$ for $15 \mathrm{~min}\left(4^{\circ} \mathrm{C}\right)$. The supernatant is brought to $60 \%$ saturation with $\mathrm{AS}$, let on ice for at least $30 \mathrm{~min}$ and centrifuged as previously. The pellet is dissolved in $1 \mathrm{~mL}$ of extraction buffer and desalted on a PD-10 column previously equilibrated with $25 \mathrm{mM}$ Tris- $\mathrm{HCl}, \mathrm{pH} 7.50$ (Tris-buffer) (fraction name: AS40-60). Ammonium sulphate is detected using Nessler's reagent in a 96-well plate. In each well $10 \mu \mathrm{L}$ of fraction, $100 \mu \mathrm{L}$ distilled water and $10 \mu \mathrm{L}$ of Nessler's reagent are introduced. This detection can be combined with the LDH screening assay.

Anion Exchange Chromatography. The desalted solution is applied to a DEAE-Sepharose CL-6B column $(2 \mathrm{~mL})$ previously equilibrated with Tris-buffer. The column is washed with 8 vol. of Tris-buffer containing $50 \mathrm{mM} \mathrm{NaCl}$, and then eluted with 8 vol. of Tris-buffer containing $0.25 \mathrm{M}$ $\mathrm{NaC}$. Two $\mathrm{mL}$ fractions are collected and aliquots screened for protein content and LDH activity. The active fractions from the DEAE column are pooled and brought to $75 \%$ saturation with AS, let on ice for at least $30 \mathrm{~min}$ and centrifuged as previously. The pellet is dissolved with $1 \mathrm{~mL}$ of Tris-buffer, and desalted on the PD-10 column (fraction name: DEAE).

Pseudo-affinity chromatography. The desalted solution is applied to a Cibacron Blue 3GA-agarose column (1 mL of gel) previously equilibrated with $12 \mathrm{~mL}$ of $10 \mathrm{mM}$ Tris- $\mathrm{HCl}$, $\mathrm{pH}$ 7.50. After washing the column with the same buffer, the active fractions (fraction name: PE) were eluted with $5 \mathrm{mM}$ $\mathrm{NADH}$ containing buffer and finally washed by $3 \mathrm{M} \mathrm{NaCl}$ containing buffer. The NADH in the active fractions was removed in a PD-10 column equilibrated with Tris-buffer.

\subsection{Protein and Activity Content of the Fractions}

Protein screening. During the chromatographic separations the protein elution was monitored by the absorbance at $280 \mathrm{~nm}\left(\mathrm{~A}_{280}\right)$ of a dilution of $40 \mu \mathrm{L}$ of each fraction in $1500 \mu \mathrm{L}$ of buffer thus using only 2 or $4 \%$ of the total fraction, depending on the step.

Protein content determination. The protein concentration 
was determined by the Lowry method [5] using several dilutions of each fraction. The standards ranged from 0 to 80 $\mu \mathrm{g}$ of bovine serum albumin per $\mathrm{mL}$ of Tris-buffer.

LDH screening assay (spot test). This quick evaluation of the presence of $\mathrm{LDH}$ activity using a 96-well plate was adapted from [6]. Creation of $\mathrm{NADH}$ in the presence of LDH drives the successive reduction of phenazine methosulphate (PMS) and then p-iodo nitrotetrazolium (INT). Ten $\mu \mathrm{L}$ of the indicated fraction and $100 \mu \mathrm{L}$ of master mix were added in each well in a 96-well plate. The master mix was prepared as follows per well: $75 \mu \mathrm{L}$ of $25 \mathrm{mM}$

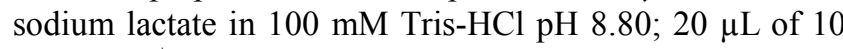
$\mathrm{mM} \mathrm{NAD}^{+} ; 5 \mu \mathrm{L}$ of $2 \mathrm{mM}$ INT; $2 \mu \mathrm{L} 2 \mathrm{mM}$ PMS. NAD+, INT and PMS should be made in distilled water and can be stored at $-20^{\circ} \mathrm{C}$ before use.

Standard LDH assay. The assay mixture, directly made in a $3 \mathrm{~mL}$ cuvette, contains $1.8 \mathrm{ml}$ of Tris-buffer; $1.0 \mathrm{ml}$ of 1.8 $\mathrm{mM}$ sodium pyruvate; and $0.1 \mathrm{ml}$ of $5 \mathrm{mM} \mathrm{NADH}$. The reaction is initiated by the addition of $0.1 \mathrm{ml}$ of diluted enzyme from the different purification steps and the decrease in $\mathrm{A}_{340}$ as a function of time is monitored. The enzyme is diluted in Tris buffer until a reasonable rate is obtained (0.1-0.2 absorbance units per min). The data set is considered as acceptable only if an $R^{2}$ of 0.99 or higher is obtained. All the assays are performed in triplicate at the laboratory temperature. Assuming a molar extinction coefficient of $6220 \mathrm{M}^{-1} \mathrm{~cm}^{-1}$ for $\mathrm{NADH}$, the change in absorbance expected for the disappearance of $1 \mu \mathrm{mol}$ of $\mathrm{NADH}$ in $3.0 \mathrm{ml}$ was taken as 2.0733 [2].

\subsection{Electrophoresis}

Discontinuous polyacrylamide gel electrophoresis [7] in Tris-HCl 25 mM, glycine 192 mM pH 8.30 (Tris-Glycine buffer) was carried out under non-denaturing conditions using $7.5 \%$ polyacrylamide and under denaturing conditions using $15 \%$ polyacrylamide. The SeeBlue ${ }^{\circledR}$ Plus2 Pre-stained Standard was used as the protein standards (phosphorylase, $148 \mathrm{kDa}$; bovine serum albumin, $98 \mathrm{kDa}$; glutamic dehydrogenase, $64 \mathrm{kDa}$; alcohol dehydrogenase, $50 \mathrm{kDa}$; carbonic Anhydrase, 36; myoglobin red, $22 \mathrm{kDa}$; lysozyme, $16 \mathrm{kDa}$; aprotinin, $6 \mathrm{kDa}$ ).

The gels were stained for proteins with the ProteoStain reagent or specifically for LDH using the PMS/INT system: $75 \mathrm{mM}$ Tris-HCl pH 8.50/1M sodium lactate/staining reagent; $8 / 4 / 2$ (vol./vol.). The staining reagent (10 $\mathrm{mg} \mathrm{NAD}^{+}$, $4 \mathrm{mg}$ INT and $1 \mathrm{mg}$ PMS in $2 \mathrm{~mL}$ of water) is prepared just before use.

\subsection{Kinetics}

\subsubsection{Pyruvate as the Substrate}

Standard procedure. The assay mixture contains $1.8 \mathrm{ml}$ of Tris buffer; $1.0 \mathrm{~mL}$ of selected sodium pyruvate concentrations, ranging from 0.02 to $15.0 \mathrm{mM}$, and $0.1 \mathrm{ml}$ of $5 \mathrm{mM}$ NADH. The reaction is initiated by the addition of 0.1 $\mathrm{ml}$ of purified enzyme, diluted such as the assay proceeds with a reasonably fast rate.
Inhibition by sodium oxamate and sodium oxalate. For the inhibition study $0.1 \mathrm{ml}$ of sodium oxamate (or sodium oxalate) at selected concentrations is substituted for an equivalent volume of Tris buffer.

Product inhibition by lactate. The assay mixture contains $0.8 \mathrm{~mL}$ of buffer, $1.0 \mathrm{~mL}$ of two or three selected pyruvate concentrations, so that no inhibition by pyruvate is visible, 1 $\mathrm{mL}$ of selected sodium L-lactate concentrations, ranging from 0.6 to $300 \mathrm{mM}$ and $0.1 \mathrm{ml}$ of $5 \mathrm{mM} \mathrm{NADH}$.

\subsubsection{Lactate as the Substrate}

Standard procedure. The assay mixture contains $1.8 \mathrm{~mL}$ of $100 \mathrm{mM}$ Tris- $\mathrm{HCl} \mathrm{pH} \mathrm{8.80,1.0} \mathrm{mL} \mathrm{of} \mathrm{selected} \mathrm{sodium}$ L-lactate concentrations, ranging from 0.6 to $300 \mathrm{mM}$, and $0.1 \mathrm{~mL} 150 \mathrm{mM} \mathrm{NAD}{ }^{+}$. The reaction is initiated by the addition of $0.1 \mathrm{ml}$ of purified enzyme, diluted such as the assay proceeds with a reasonably fast rate. Where necessary the buffer contained $350 \mathrm{mM}$ hydrazine hydrate.

Product inhibition by pyruvate. The assay mixture contains $0.8 \mathrm{~mL}$ of $100 \mathrm{mM}$ Tris- $\mathrm{HCl} \mathrm{pH} 8.80,1.0 \mathrm{~mL}$ of selected sodium pyruvate concentrations, ranging from 0 to $0.5 \mathrm{mM}, 1.0 \mathrm{~mL}$ of two or three selected L-lactate concentrations, so that no inhibition by L-lactate is visible, and $0.1 \mathrm{~mL} 150 \mathrm{mM} \mathrm{NAD}^{+}$.

\section{Results and Discussion}

The results presented thereafter are representative of the data-sets obtained by the student-pairs and are comparable to those obtained by the instructors and the reference publications. Unless noted otherwise the variability from one student-pair to the other was not significant.

\subsection{LDH Screening Assay (Spot Test)}

This quick detection of the LDH activity is possible after incubation at RT, and the appearance of purple colour in a well indicates a significant amount of LDH activity in that fraction. The lower limit of sensitivity for the assay is about $0.01 \mathrm{U}$ of LDH activity per well that are needed to produce any colour change visible by eye after a few min incubation. The plates are photographed when the colour development is sufficient. If available, the course of colour development can also be monitored on a plate reader at $\mathrm{A}_{520}$. Alternately the reaction can be stopped by the addition of $100 \mu \mathrm{L} 9 \mathrm{M}$ urea. The high concentrations of urea denature the enzyme, resulting in a complete quench of the reaction and urea does not interfere with the colour intensity.

\subsection{Purification of Pig Heart $\mathrm{LDH}$}

The purification described here was started using approximately $50 \mathrm{~g}$ of pig heart with $100 \mathrm{~mL}$ extraction buffer which is enough for up to 8 student laboratory pairs. Each student-pair begins with $10 \mathrm{~mL}$ of the CE fraction.

The purification steps chosen (Table 1), namely AS fractionation, anion exchange chromatography, and affinity chromatography are techniques widely used for enzyme purification. 
Table 1. Purification scheme

\begin{tabular}{lll}
\hline Step & Procedures performed & $\begin{array}{l}\text { Name of the } \\
\text { fraction }\end{array}$ \\
\hline 1 & $\begin{array}{l}\text { Preparation of the crude extract } \\
2\end{array}$ & $\begin{array}{l}\text { Ammonium sulphate (AS) fractionation } \\
\text { Desalting on PD-10 column }\end{array}$ \\
& $\begin{array}{l}\text { Anion exchange chromatography } \\
\text { Concentration of the DEAE fractions } \\
\text { Desalting/buffer exchange on PD-10 column }\end{array}$ & AS40-60 \\
4 & \begin{tabular}{l} 
Pseudo-affinity chromatography \\
\hline
\end{tabular}
\end{tabular}

Concerning the AS fractionation, a saturation between 40 and $60 \%$ was selected (fraction AS40-60) since the LDH activity is essentially recovered in this fraction. The pellet obtained at $40 \%$ displayed low activity and was discarded together with the final supernatant which contains a significant amount of activity but also proteins that could interfere in the subsequent purification steps. As a proof when the second precipitation was done at $70 \%$ saturation, instead of $60 \%$, the corresponding AS40-70 fraction was markedly coloured in red, probably due to haemoglobin, and displayed in average $10 \%$ less activity, and 30\% less specific activity.

After desalting on the PD-10 column, the active fractions were subjected to the anion exchange chromatography step (Figure 1). No activity was found in the fractions eluted with the Tris buffer containing $50 \mathrm{mM}$ (arrow 'a') and $500 \mathrm{mM}$ $\mathrm{NaCl}$ (arrow 'c') respectively. The activity was mainly eluted with the buffer containing $250 \mathrm{mM} \mathrm{NaCl}$ (arrow 'b').

The active fractions from DEAE (21 to 25) were concentrated by AS precipitation and then desalted on the PD-10 column in order to prepare the subsequent step, sensitive to the presence of salts. As shown in Figure 2, the monitoring of the AS with the Nessler's reagent help to take the decision on the choice of the fractions for the following step. In the present example, fractions 4 to 6 were selected and it was shown that the AS amount present in fraction 6 , less than $25 \mu \mathrm{g} / \mathrm{mL}$, did not interfered with the pseudo-affinity step.

The major part of the activity (80\%) was recovered in the fraction eluted from the Cibacron blue 3GA-agarose column with the phosphate buffer containing $5 \mathrm{mM}$ NADH (Figure $3 \mathrm{~A}$, arrow 'a'). A low amount of activity is visible at the beginning of the chromatography, and this was also seen even if fraction 6 from the PD10 step was omitted. It is actually possible to detect LDH with the spot test for the first 12 fractions (Figure 3B), but due to the presence of NADH, only the use of the standard LDH assay is possible to measure the enzyme activity in those fractions.

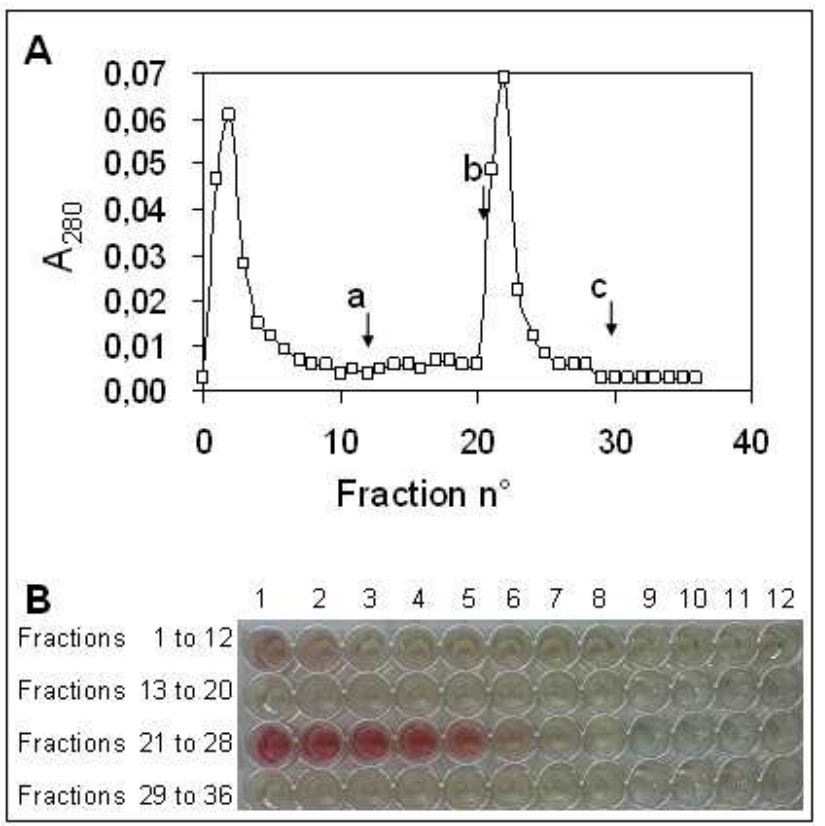

Figure 1. DEAE anion exchange chromatography step. After the application of the sample, the column was eluted (2 $\mathrm{mL}$ fractions) by a discontinuous salt gradient in Tris-buffer: With $25 \mathrm{mM} \mathrm{NaCl}$, fractions 1-12; $50 \mathrm{mM} \mathrm{NaCl}$, arrow ' $a$ ', fractions 13-20; $250 \mathrm{mM} \mathrm{NaCl}$, arrow ' $b$ ' fractions 21-28; and $500 \mathrm{mM} \mathrm{NaCl}$, arrow 'c', fractions 29-36. The fractions were assayed for total protein $(A)$ and for LDH activity with the spot test $(B)$.

Since this purified enzyme is intended for kinetic studies using L-lactate as the substrate a final PD-10 chromatography step is mandatory to remove traces of $\mathrm{NADH}$. This is done as for the desalting step.

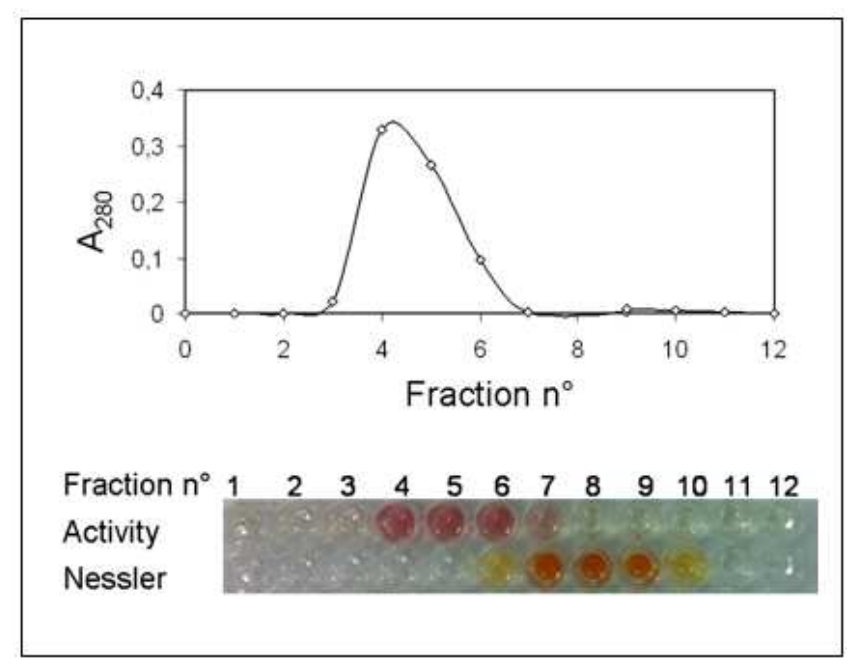

Figure 2. Desalting of the active fractions from DEAE. Lane 1: the fractions containing the activity are monitored with the spot test Lane 2: detection of AS using the Nessler's reagent. 


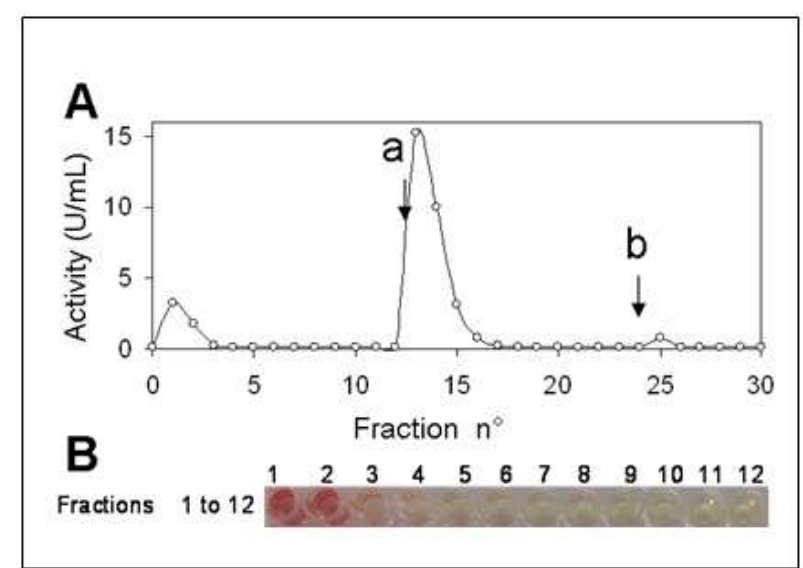

Figure 3. Pseudo-affinity chromatography step. After the application of the sample the column was washed with $10 \mathrm{mM}$ Tris- $\mathrm{HCl}, \mathrm{pH} 7.50$ and eluted with the same buffer containing $5 \mathrm{mMNADH}$ (arrow' ')' and finally washed with buffer containing $3 \mathrm{M} \mathrm{NaCl}$ (arrow ' $b$ '). (A) The activity was measured with the standard LDH assay. (B) The spot test was only used for fractions 1-12 due to the presence of NADH in other fractions.

\subsection{Characterisation of Pig Heart LDH}

\subsubsection{Protein and Activity Content of the Fractions}

The intent of the purification scheme (Table 1) was not to obtain a preparation of the highest specific activity with the maximum percent yield, but instead to adequately illustrate the major techniques in the "built-in" project utilizing a single enzyme, LDH.

As shown in Table 2, the AS cut chosen (40-60\%) sacrifices a large percent of the activity $(>40 \%)$. The choice of scaling-down the purification process - starting from the equivalent of $5 \mathrm{~g}$ of pig heart per student-pair, and using small size columns - is also responsible of significative loss of material. In any purification step there is a trade-off between purity and yield. The losses are lower for the subsequent steps ( $25 \%$ per step). In terms of improvement of the specific activity, the key step is the pseudo-affinity chromatography. The resulting $\mathrm{PE}$ fraction is adequate and sufficient for the kinetics study. When the purification process was carried out with the AS40-70 fraction, 25\% less activity was recovered in the final step, the specific activity being however equivalent to the one obtained from AS40-60.

In the case where the purpose of a practical work is to rapidly prepare heart LDH in view of kinetic studies, it is possible to shorten the purification scheme by omitting the DEAE chromatography step without notable decrease in the purification level.

\subsubsection{Characterisation of the Fractions by Electrophoresis}

The fractions obtained in the different purification steps were subjected to electrophoresis under non-denaturing conditions in order to visualize in parallel for each fraction, the proteins detected by the protein stain (Figure 4A) and by the LDH-specific stain (Figure 4B). The obtained profiles are in good correlation with the degree of purification and the yield (Table 2).
Table 2. Purification of LDH, starting from the equivalent of $5 \mathrm{~g}$ of pig heart (10 $\mathrm{mL}$ of crude extract).

\begin{tabular}{cccccc}
\hline Fraction & $\begin{array}{c}\text { Activity } \\
\text { (U) }\end{array}$ & $\begin{array}{c}\text { Protein } \\
(\mathbf{m g})\end{array}$ & $\begin{array}{c}\text { Specific } \\
\text { activity } \\
\text { (U/mg) }\end{array}$ & $\begin{array}{c}\text { Purifica- } \\
\text { tion (fold) }\end{array}$ & $\begin{array}{c}\text { Yield } \\
(\mathbf{\%})\end{array}$ \\
\hline $\mathrm{CE}$ & 87.51 & 178.75 & 0.49 & 1 & 100 \\
$\mathrm{AS} 40-60$ & 51.37 & 48.42 & 1.06 & 2.16 & 58.70 \\
$\mathrm{DEAE}$ & 38.10 & 9.54 & 3.99 & 8.15 & 43.53 \\
$\mathrm{PE}$ & 29.08 & 1.08 & 26.93 & 54.96 & 33.23 \\
\hline
\end{tabular}

The LDH-specific visualization system helps identifying which of the protein bands contains LDH. Heart tissue primarily contains the B4 isoenzyme, but also smaller amounts of the other four isoenzymes AB3, A2B2, A3B and $\mathrm{A} 4$ are revealed, the higher the proportion of the $\mathrm{B}$ subunit, the faster the electrophoretic mobility (Wolf 1988). The B4 isoenzyme is thus clearly identified in Figure 4B, lane 4 , and by reference in all the other lanes. By the way it is noticed that the profile is simplified for the purified enzyme, displaying almost exclusively the B4 isoenzyme. This was emphasised by the fact that a high L-lactate concentration used in the staining reagent favours the staining of the A-containing $\mathrm{LDH}$ isoenzymes and especially LDH A4 [8].

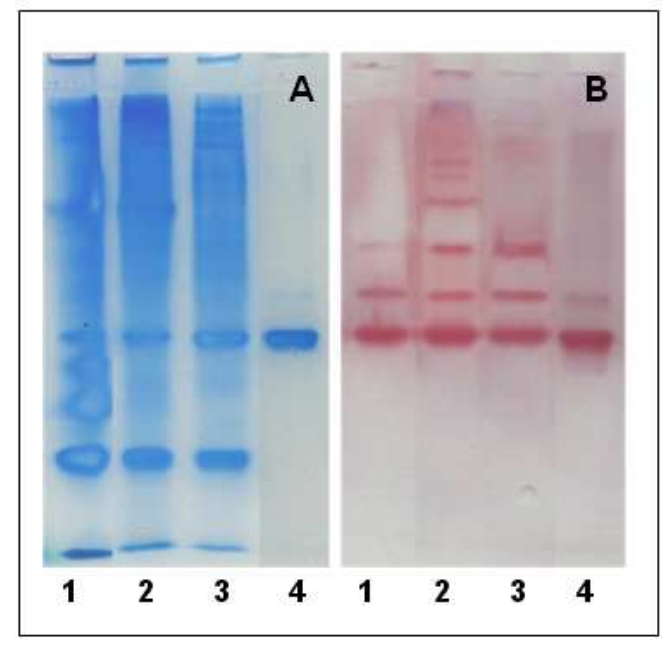

Figure 4. Electrophoresis under non-denaturing conditions $(7.5 \%$ and $4.2 \%$ polyacrylamide for separation and stacking gels respectively) of the fractions obtained during the purification of porcine heart $L D H(A)$ Proteins were revealed with ProteoStain. (B) LDH was revealed using the specific visualisation system of its activity. Equal protein amounts of each fraction $(10 \mu \mathrm{g})$ obtained during purification were used; lane 1: CE; lane 2: SA40-60; lane 3: DEAE; lane 4: PE.

The SDS-PAGE (Figure 5) confirms the importance of the pseudo-affinity chromatography step and the gap in the specific activity seen in Table 2 for the PE fraction. The homogeneity of the purified fraction is also confirmed, only few minor bands are visible on the gel. The LDH monomer band is near $36 \mathrm{kDa}$ which is in agreement with the value calculated from the amino acid composition, 334 amino acids, 36,612 Da (Uniprot, http://www.uniprot.org accession $\left.\mathrm{n}^{\circ} \mathrm{P} 00336\right)$. 


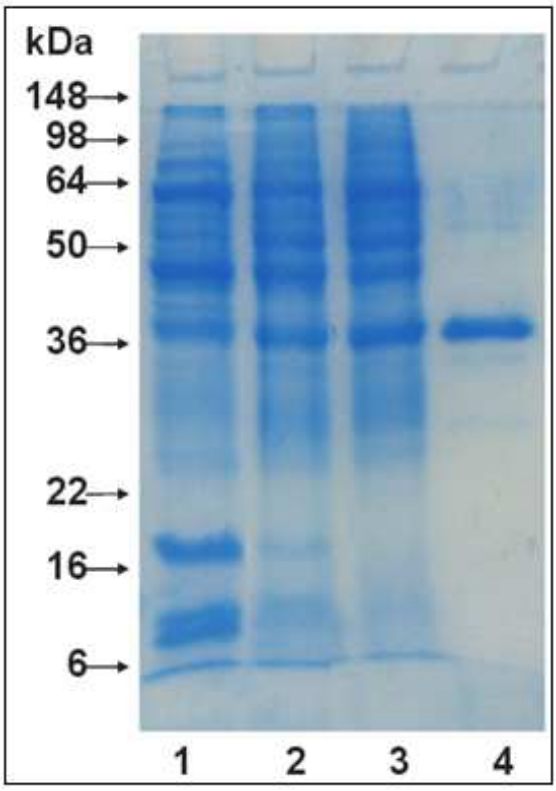

Figure 5. Electrophoresis under denaturing conditions (15\% and $4.2 \%$ polyacrylamide for separation and stacking gels respectively) of the fractions obtained during the purification of porcine heart LDH. Proteins were revealed with ProteoStain. Equal amounts of each fraction obtained during purification were used. lane 1: CE; lane 2: SA40-60; lane 3: DEAE, lane 4: PE. The position and mass of the standard proteins ware indicated at left.

In view of all the results it appears that the purified $\mathrm{LDH}$ from pig heart correspond to the B4 isoenzyme and thus it will be referred to thereafter in the text as such for the kinetics study.

\subsection{Kinetics of the Purified LDH}

The kinetic studies are performed in both directions using pyruvate or lactate as the substrate. This allows illustrating several aspects of enzyme kinetics including the potential inhibition by substrates and products.

\subsubsection{Pyruvate as the Substrate}

In the first phase of the experiment students have to work with a large range of pyruvate concentrations, up to $5 \mathrm{mM}$, the instruction being to use several points below $1 \mathrm{mM}$ in view of a better estimate of the $\mathrm{Km}$ value. A representative data set is shown in Figure 6. The inhibitory effect was even grater for several student-pairs depending on the LDH in the assay (not shown). The students thus find out that substrate inhibition occurs. This is a starting point to make them search what is the in vivo situation and in particular what are the physiological concentrations of the substrate. In vitro, even higher levels of substrate pyruvate inhibition were reported for the B4 isoenzyme, up to $70 \%$ at $5 \mathrm{mM}$ [9] but what could be the in vivo significance when e.g. after $30 \mathrm{~s}$ of tetanus the level of pyruvate attains $0.84 \mathrm{mM}$ ? From the plots good estimates could be calculated for $\mathrm{K}_{\mathrm{m}}$ and $\mathrm{V}_{\max }$. The $\mathrm{Km}$ values, around 0.1-0.2 $\mathrm{mM}$, were close to the previously published ones, while Vmax varied with the enzyme concentration.

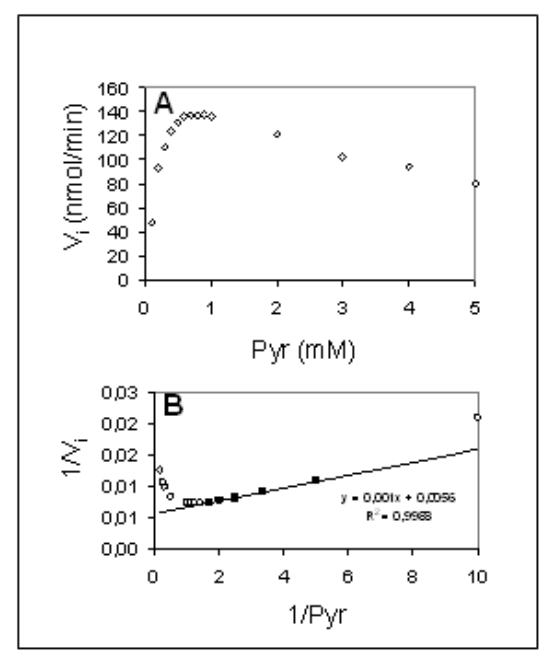

Figure 6. Kinetic analysis of the purified heart LDH B4 with pyruvate as the substrate. (A) Michaelis-Menten and (B) Lineweaver-Burk plots of a representative data-set. The purified enzyme was assayed from 0.1 to 5 $m M$ pyruvate. The black squares indicate the points retained for Vmax and Km calculations.

In the second phase of the experiments the students have to evaluate the effect of sodium oxamate. To avoid the superimposition of the effect of pyruvate as a potential inhibitor, they are asked to select low concentrations of pyruvate. In the example presented in Figure 7 the effect of oxamate as a competitive inhibitor is visible even though inhibition by pyruvate seems still visible over $0.4-0.5 \mathrm{mM}$ pyruvate concentrations. The experiment was also done with sodium oxalate (not shown) in order to demonstrate its action as a non-competitive inhibitor. The students are asked to compare the structures of oxamate and oxalate to those of pyruvate and lactate to find-out the structural homologies and conclude as to their inhibitor effects.
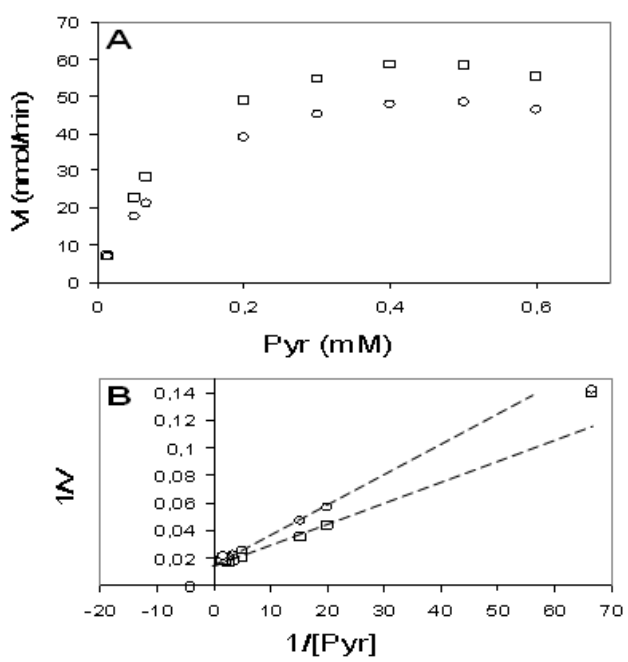

Figure 7. Kinetic analysis of the purified heart $L D H B 4$ with pyruvate as the substrate and effect of sodium oxamate. (A) Michaelis-Menten and (B) Lineweaver-Burk plots of a representative data-set. The purified enzyme, $0.1 \mathrm{U}$, was assayed from 0.01 to $0.6 \mathrm{mM}$ pyruvate in the absence ( $\square$.) and presence of $0.1 \mathrm{mM}$ oxamate $(\mathrm{O})$. 


\subsubsection{L-lactate as the Substrate}

As the use of a high $\mathrm{pH}$ buffer favours the reaction in the direction of pyruvate production [3] $100 \mathrm{mM}$ Tris- $\mathrm{HCl} \mathrm{pH}$ 8.80 was used for this experiment to decrease the potential inhibitor effect of L-lactate at high concentrations. The enzymatic activity was plotted as the percentage of the maximal activity against the final L-lactate concentration to allow comparison with the plot in the presence of hydrazine (Figure 8).

Hydrazine favours a second fast, non enzymatic reaction that converts pyruvate to pyruvate-hydrazone. Pyruvate-hydrazone cannot bind as well as pyruvate to the active site of LDH, thus, the coupled reaction prevents reversibility and also decreases the likelihood of product inhibition [10]. This is clearly seen in Figure 8 for concentrations over $20 \mathrm{mM}$ L-lactate.

The use of hydrazine is interesting in order to explain to the students the in vivo situation. In fact the pyruvate can potentially be used by another enzyme and thus prevent any inhibitory effect.

\subsection{Comparative Kinetics of the Purified Heart LDH B4 and a Commercial Muscle LDH A4}

This comparative study was designed to initiate students with the possibility that different isoforms of a given enzyme could display particular kinetic properties which could potentially reflect their function. This is an important issue as in the past it appeared to be some confusion in the textbooks concerning the physiological significance of why different tissues possess their particular complement of isoenzymes $[4,11]$.

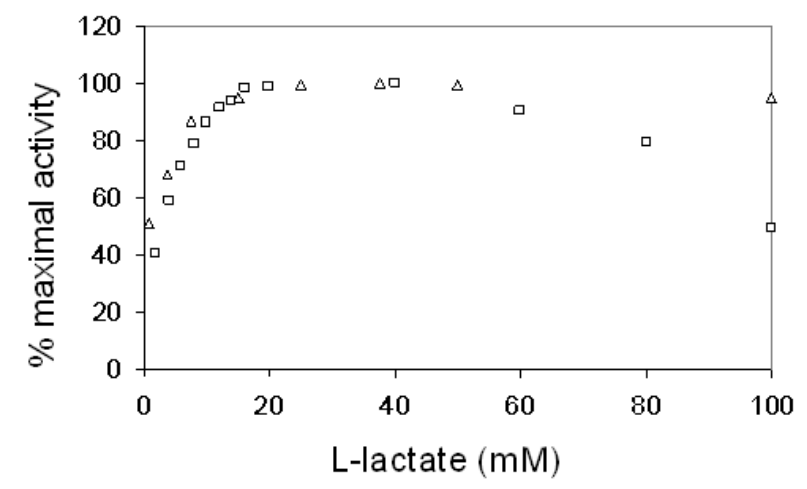

Figure 8. Kinetic analysis of the purified heart LDH B4 with L-lactate as the substrate. The enzymatic activity was plotted as the \% of the maximal activity against the final L-lactate concentration. Plot of a representative data-set. Aliquots of the purified enzyme from the pseudo-affinity chromatography purification step were assayed from 2 to $100 \mathrm{mM}$ L-lactate in the absence $(\square$ and presence ( $\Delta$ ) of $200 \mathrm{mM}$ hydrazine.

\subsubsection{Pyruvate as the Substrate}

The maximal activity for the purified B4 isoenzyme was reached at lower pyruvate concentrations than for the A4 isoenzyme (Figure 9). Under the experimental conditions used, no inhibition was visible for the A4 isoenzyme in contrast to the B4 isoenzyme.

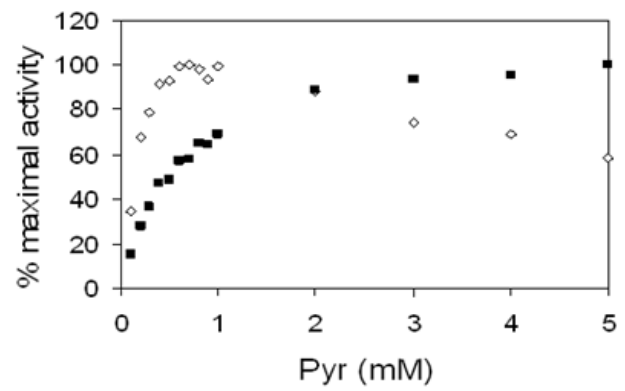

Figure 9. Effect of pyruvate substrate concentration. on the kinetic properties of the purified heart $\mathrm{LDH} B 4,(\nabla)$, and the commercial muscle LDH A4 (-) isoenzyme. The enzymatic activity was plotted as the \%of the maximal activity against the final pyruvate concentration. Aliquots of he enzymes were assayed from 0.1 to $5 \mathrm{mM}$ pyruvate.

The inhibition of LDH by its substrate pyruvate was discussed in the past $[4,11]$ and we were aware of this situation which is most likely due to the low enzyme and pyruvate concentrations used in the in vitro assays. Indeed, calculations revealed that 'in vivo LDH exists in concentrations several orders of magnitude above those for the spectrophotometric assay in which pyruvate inhibition occurs' [12].

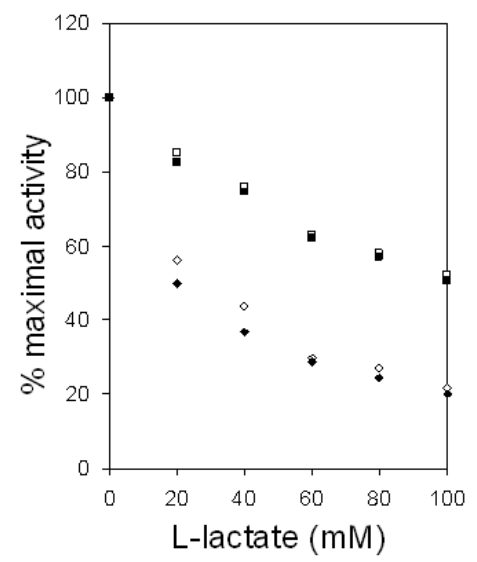

Figure 10. Product inhibition by L-lactate. Comparison of the kinetic properties of purified heart LDH B4 and the commercial muscle LDH A4 with pyruvate as the substrate $(\diamond 0.15 \mathrm{mM}$ and $\$ 0.25 \mathrm{mM}$ for $B 4 ; \square 0.3$ $\mathrm{mM}$ and $0.5 \mathrm{mM}$ for A4). The enzymatic activity was plotted as the \%of the maximal (uninhibited) activity against the final lactate concentration. Aliquots of the enzymes were assayed from 0 to $100 \mathrm{mM}$ L-lactate.

It has been suggested that an abortive ternary complex composed of LDH, pyruvate, and NAD may be responsible for the pyruvate inhibition of dilute concentrations of $\mathrm{LDH}$. The abortive ternary complex forms very slowly at physiologic LDH concentrations [13]. It was reported that at $25^{\circ} \mathrm{C}$ LDH B4 displayed no inhibition by concentrations of pyruvate up to $20 \mathrm{mM}$ when assayed at concentrations of enzyme of $4 \times 10^{-7} \mathrm{M}$ and upwards [12], concentrations that cannot be used under the experimental conditions in the practical work described here (unavailability of a stopped-flow apparatus).

Product inhibition of LDH by L-lactate was effective for both isoenzymes with different levels (Figure 10). At $20 \mathrm{mM}$ 
L-lactate, concentration considered as physiological in muscles, the A4 isoenzyme displayed less than $20 \%$ inhibition in contrast to B4 which displayed near $50 \%$ inhibition.

\subsubsection{L-lactate as the Substrate}

The results of a study on the product inhibition of LDH by pyruvate (Figure 11) demonstrated that the B4 isoenzyme is inhibited approximately $40 \%$ by a concentration of $0.13 \mathrm{mM}$ pyruvate reported to occur in contacting muscles, not much different to that of the A4 isoenzyme for which the inhibition extend is approximately $35 \%$ and comparable to published values [9].
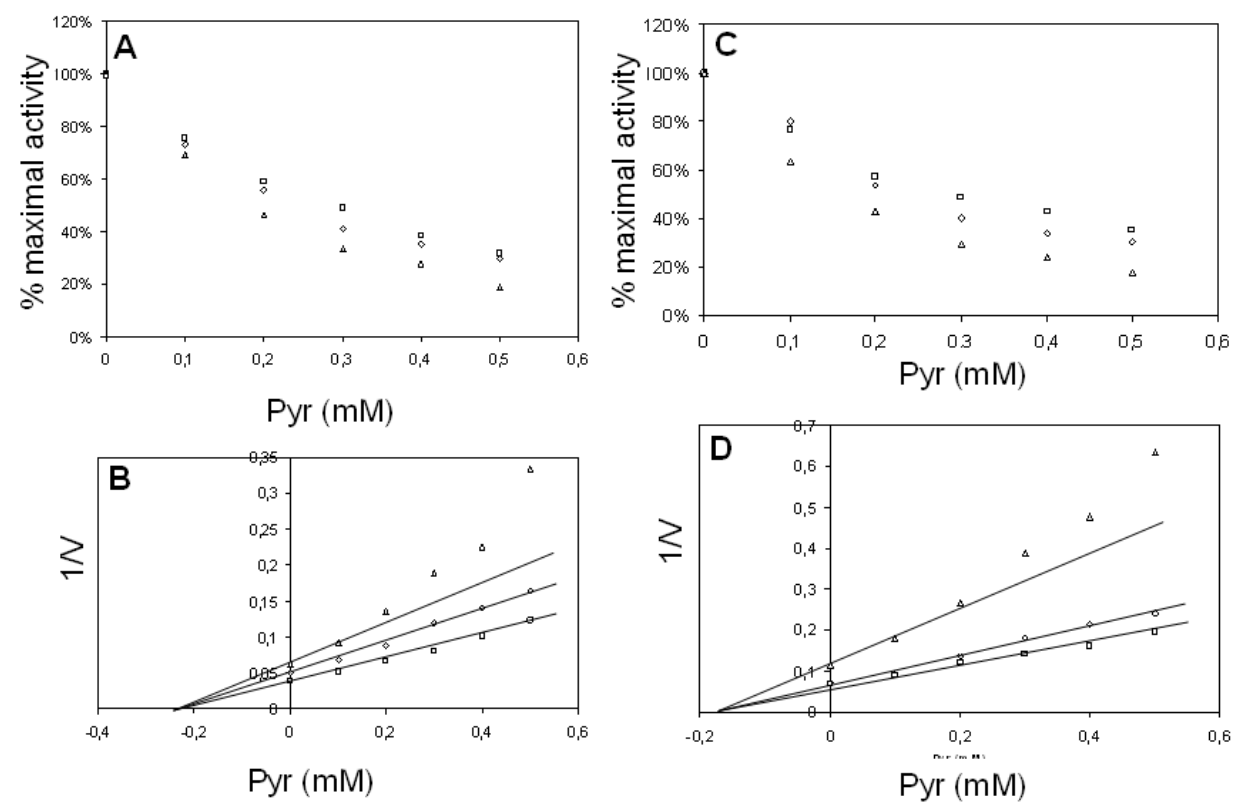

Figure 11. Product inhibition by pyruvate. The enzymatic activity was plotted as the percentage of the maximal activity against the final pyruvate concentration $(A$ and $C$ ) and as Dixon plots. The reciprocal of the initial velocity (nmol/min) was plotted against the concentration of pyruvate. Aliquots of the heart LDH B4 (A and B) and of muscle LDH A4 (C and D) were assayed from 0 to $0.5 \mathrm{mM}$ pyruvate in the presence of $3 \mathrm{mM}(\Delta), 6 \mathrm{mM}(\nabla)$ and $10 \mathrm{mM}$ $(\square$ L-lactate for $\mathrm{LDH} B 4$ and $10 \mathrm{mM}(\Delta), 20 \mathrm{mM}(\diamond)$ and $40 \mathrm{mM}(\square)$ L-lactate for $\mathrm{LDH} A 4$

\subsection{Technical Remarks - Pitfalls}

The purification scheme used in the presented practical work was designed for the isolation of $\mathrm{LDH}$ from pig heart. Depending on the origin of the tissue used, this scheme can be adapted by making reference to the general procedures that have been used for the purification of LDH from a number of vertebrates [14]. The size of the columns and the volumes of gels used for ion exchange and pseudo-affinity chromatography can be adapted proportionally to the initial quantities used.

A friendly ammonium sulphate calculator, that takes into account the working temperature, is available at http:/www.encorbio.com/protocols/AM-SO4.htm.

It is noteworthy that the enzyme assays were carried out in the laboratory temperature conditions and that one should take into account that the obtained results could vary in relation to the period of the year the experiments are carried-out. In our hands, the first part of the work (Purification/characterisation/kinetics; 6 days) take place during winter, with temperatures that average $18-20{ }^{\circ} \mathrm{C}$ in the laboratory. Higher activity levels were measured during the second part of the work (comparative kinetics) which takes place at spring, with higher temperatures, $22-25^{\circ} \mathrm{C}$.

For electrophoresis, in order to minimise risks, a solution of $30 \%$ acrylamide/bis-acrylamide $(2.7 \%$ cross-liker $)$ was used. For protein staining in polyacrylamide gels, Coomassie Brilliant Blue can be used [15] instead of ProteoStain.

One of the typical student pitfalls that might be encountered in the laboratory is the improper use of micropipettes causing distortion of the obtained values for the assays. The instructor should be aware of that and do not hesitate to ask the students to verify their ability in using micropipettes and also verify the use of the proper size micropipette and tip.

\subsection{Student Expected Data Analysis and Discussion}

The students are asked to produce a write-up presented as a publication in a biochemistry journal that is organised in three parts, namely (i) an 'introduction', (ii) a 'results and discussion' section, with a selection of the conclusive data sets, adequate layout for figures and tables and well documented legends, and (iii) a 'conclusion'. The main text cannot exceed 10,000 characters. It is not allowed to merely reproduce the handout distributed during the pre-lab lecture 
- containing the background and the experimental section but students are allowed to add as an appendix all the obtained data and notes they want.

\section{Teaching Points}

From the pedagogical point of view, an enzyme purification and characterisation project has two objectives. First, to initiate students to a variety of techniques, which are widely used in biochemistry. Second, to demonstrate during the continuous laboratory period a number of fundamental biochemical principles since each technique exploits a different biochemical property of the enzyme. This practical work is currently organised in two distinct parts, the first at the end of the winter period (6 days), the second at the end of the spring period ( 3 days).

To prepare the first part of the practical work, a pre-lab lecture takes place during the semester, a few weeks before the session starts, so that the students have time to read the handout containing the background information and the experimental section. Two laboratory days are needed for the purification (section 4.2) and three more for the characterisation (4.3). An additional day is necessary for a first kinetic study of the purified enzyme (section 4.4.1).

For the second part of the practical work three laboratory days are necessary and no preparation is needed for the students during the semester, the project should be discovered by them at the beginning of the session. They are asked to use their knowledge from the first part of the work in order to propose a series of experiments to address the comparison of the kinetic properties of two enzymes: (i) the one they have purified from pig heart; and (ii) the commercial muscle LDH. Half of the student-pairs will work with one of the isoenzymes. Each student-pair has to evaluate the possible inhibition by pyruvate (or L-lactate) as the substrate and as the product, which mean 4 distinct experiments. The instructor has to verify the feasibility of each proposed protocol and help them start. At the end of the work the different student-pairs have to introduce their results to the others. This step enables the comparison of the kinetic properties of the two isoenzymes.

An aspect that students particularly appreciate is the fact that we ask them to produce a write-up presented as a publication in a biochemistry journal. We consider this as a real added value to their education.

Many of the general skills, procedures, principles and methods considered to contribute to teaching a modern biochemistry laboratory [16] were used in the undergraduate biochemistry laboratory experiment described here.

One of the prominent points of the overall approach is the training and the compliance to the safety regulations in the biochemistry laboratory. And this is the reason why one of the learning outcomes covers this aspect. The instructor has an important role for this aspect. At the end of the first part of the work the student should be confident with reading and applying the instructions in the Safety Data Sheets (MSDS) and in particular concerning section 8: Exposure Controls/Personal Protection. The MSDS of all the products used during the work are grouped in a specific file which is continuously up-dated at the student's disposal in the laboratory.

\section{Concluding Remarks}

The purpose of the present paper was to describe the achievement of a practical work that several generations of undergraduate students have practiced. This practical work was designed in the aim of combining a number of independent laboratory periods, and tries to mimic a "research laboratory" environment. Thanks to that the students have a better understanding of the constraints in the biochemistry laboratory. They prepare themselves all the buffers and reagents they need and are trained to organise and perform the experiments timely and efficiently while being aware of the safety regulations.

The reader will find herein detailed information for reproducing the experiments. The purification scheme, the characterisation and the kinetic studies can be adapted according to the available materials and time period. One could be surprised by the fact that not many recent references are presented; the reason is that most of the recent references are dealing with the function of the enzyme and cannot help concerning the purification, characterisation and kinetics that have largely worked-out many years ago.

As it was recently pointed out [6], hands-on experience with performing experiments and analyzing data helps students to develop problem-solving skills that will benefit them throughout their life. And according to a popular editorial in Science [17] such problem-solving skills are not being adequately taught to undergraduate students, the result of too much focus on presenting scientific explanations without covering the scientific processes and reasoning.

The multistep purification of an enzyme from its source material, where students perform different purification techniques over successive laboratory days, the characterisation of the purified enzyme, and the extensive approach of enzyme kinetics, naturally fits into a project-based biochemistry learning process.

\section{Acknowledgements}

I congratulate all the instructors that over the years contributed to the success of the practical work described here and who patiently and timely applied the modifications that have been successively introduced. In particular, I wish to thank Sandrine Levasseur, technician of the biochemistry laboratory, for her continuous expert contribution. Undoubtedly I cannot forget all the moments of conviviality shared with the students I have myself supervised. 


\section{Nomenclature}

$\mathrm{A}_{\lambda}$, absorbance at the specified $\lambda$; AS, ammonium sulphate; CE, crude extract; INT, p-iodo nitrotetrazolium ; LDH, Lactate dehydrogenase; MSDS, Safety Data Sheets; PE, purified enzyme; PMS, phenazine methosulphate; Pyr, pyruvate; $\mathrm{U}$, unit of enzyme activity; $\mathrm{Vi}$, initial velocity; Vol, volume;

\section{References}

[1] A.J. Turner, A simple a colourful procedure to demonstrate the principles of affinity chromatography, Biochem. Educ., 7 (1979) 60-61.

[2] E.C. Wolf, The Partial Purification and Characterization of Lactate Dehydrogenase, Biochem. Educ., 16 (1988) 231-234.

[3] R.J. Gay, R.B. McComb, G.N. Bowers, Optimum reaction conditions for human lactate dehydrogenase isoenzymes as they affect total lactate dehydrogenase activity, Clin. Chem., 14 (1968) 740-753.

[4] K.L. Manchester, Kinetics of lactate deshydrogenase: a textbook problem, Biochem. Educ., 5 (1977) 15.

[5] O.H. Lowry, N.J. Rosebrough, A.L. Farr, R.J. Randanll, Protein measurement with the Folin phenol reagent, J. Biol. Chem., 193 (1951) 265-275.

[6] A.B. Coleman, New ideas for an old enzyme: A short, question-based laboratory project for the purification and identification of an unknown LDH isozyme, Biochem. Mol. Biol. Educ., 38 (2010) 253-260.

[7] U.K. Laemmli, Cleavage of Structural Proteins during the Assembly of the Head of Bacteriophage T4, Nature, 227 (1970) 680-685.
[8] P.H. Springell, T.A. Lynch, Staining artifacts following electrophoretic separation of LDH isozymes, Anal. Biochem., 74 (1976) 251-253.

[9] R. Stambaugh, D. Post, Substrate and product inhibition of rabbit muscle lactic dehydrogenase heart $(\mathrm{H} 4)$ and muscle (M4) isozymes, J. Biol. Chem., 241 (1966) 1462-1467.

[10] J.L. Powers, N.E. Kiesman, C.M. Tran, J.H. Brown, V.L.H. Bevilacqua, Lactate Dehydrogenase Kinetics and Inhibition Using a Microplate Reader, Biochem. Mol. Biol. Educ., 35 (2007) 287-292.

[11] K.L. Manchester, LDH: Plus ça change, plus c'est la même chose Continuing problems with textbook presentations of the kinetic properties of the isozymes of lactate dehydrogenase, Biochem. Educ., 22 (1994) 91-93.

[12] T. Wuntch, R.F. Chen, E.S. Vesell, Lactate dehydrogenase isozymes: kinetic properties at high enzyme concentrations, Science, 167 (1970) 63-65.

[13] T. Wuntch, E.S. Vesell, R.F. Chen, Studies on rates of abortive ternary complex formation of lactate dehydrogenase isozymes, J. Biol. Chem., 244 (1969) 6100-6104.

[14] A. Pesce, T.P. Fondy, F. Stolzenbach, F. Castillo, N.O. Kaplan, The comparative enzymology of lactic dehydrogenases 3 Properties of the H4 and M4 enzymes from a number of vertebrates, J. Biol. Chem., 242 (1967) 2151-2167.

[15] S. de S. Groth, R. Webster, A. Datyner, Two new staining procedures for quantitative estimation of proteins on electrophoretic strips, Biochim. Biophys. Acta, 71 (1963) 377-391.

[16] R. Boyer, Concepts and skills in the biochemistry/molecular biology lab, Biochem. Mol. Biol. Educ., 31 (2003) 102-105.

[17] B. Alberts, Redefining science education, Science, 323 (2009) 437. 